\title{
0000 Effectiveness of Retrograde Angiography Technical via the Contralateral Carotid Artery in Mechanical Thrombectomy for Carotid Non-T Occlusion by Dual Puncture Technique
}

Atsushi Uyama, Kazuma Tsuto, Takashi Matsumoto, and Masataka Takeuchi

Objective: We report the effectiveness of retrograde angiography via the contralateral carotid angiography using a dual puncture technique in mechanical thrombectomy (MT) for non-T occlusion in patients with acute internal carotid artery (ICA) occlusion not involving the ICA terminus.

Case Presentation: In the dual puncture technique, arterial puncture is performed at two sites: a balloon guiding catheter (BGC) is navigated to the ICA on the affected side and another catheter is navigated to the unaffected side. Thrombus retrieval is performed by manual aspiration through the BGC and MT using a stent retriever and/or aspiration device. Reperfusion is confirmed by retrograde angiography via the carotid artery on the unaffected side, with manual aspiration through the BGC on the affected side. Throughout the procedure, the BGC blocks the blood flow in the ICA on the affected side until reperfusion is confirmed. No distal embolization was occurred in our three patients treated using this technique. Conclusion: Application of the dual puncture technique on MT is recommended for non-T occlusion to prevent distal embolization.

Keywords mechanical thrombectomy, non-T occlusion, acute internal cerebral artery occlusion, retrograde angiography, contralateral carotid artery

\section{Introduction}

The outcome of acute internal carotid artery (ICA) occlusion remains poor, even after employing various revascularization procedures. ${ }^{1)}$ Recently, favorable results of mechanical thrombectomy (MT) were reported.,3) However, in patients with acute ICA occlusion, distal embolization may occur during MT due to substantial thrombus in a large-caliber vessel. ${ }^{4-6)}$ ICA occlusion may involve an extensive area (from the cervical portion to the terminus), but no study has examined occluded-site-related differences

Department of Neurosurgery, Seisho Hospital, Odawara, Kanagawa, Japan

Received: October 14, 2020; Accepted: January 20, 2021 Corresponding author: Atsushi Uyama. Department of Neurosurgery, Seisho Hospital, 1-16-35, Ogicho, Odawara, Kanagawa 250-0001, Japan

Email: auyama811@yahoo.co.jp

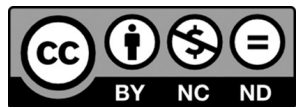

This work is licensed under a Creative Commons Attribution-NonCommercialNoDerivatives International License.

(C)2021 The Japanese Society for Neuroendovascular Therapy in treatment strategies or results. Treatment strategies depend on whether the site of occlusion involves the ICA terminus, that is, T occlusion (Fig. 1A) or non-T occluson?) (Fig. 1B). In particular, distal embolization to the ICA terminus or middle cerebral artery (MCA) in patients with non-T occlusion may lead to marked deterioration of neurological symptoms. Here, we report the effectiveness of retrograde angiography via the contralateral carotid artery using the dual puncture technique to prevent distal embolization in MT for non-T occlusion of the ICA.

\section{Case Presentation}

\section{Dual puncture technique}

In the dual puncture technique, arterial puncture is performed at two sites. In general, the bilateral femoral arteries are punctured. A balloon guiding catheter (BGC) is inserted into the affected-side ICA, and a diagnostic catheter is inserted into the unaffected side of the carotid artery. Contrast medium retention at the proximal ICA is confirmed using angiography through the BGC on the affected side (Fig. 2A). A collateral pathway to the affected side via 
A

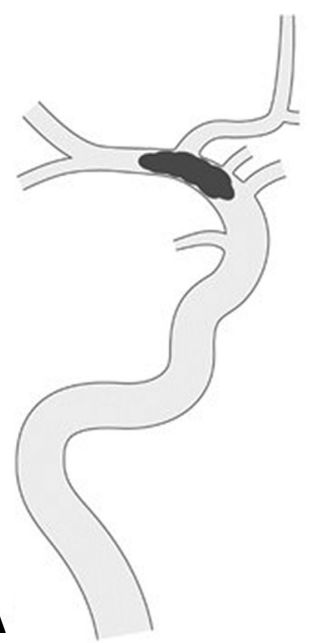

B

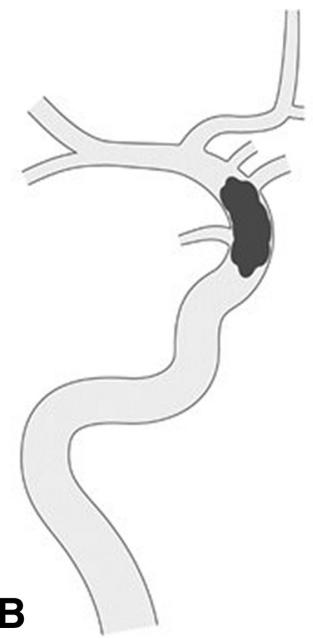

Fig. 1 (A) T occlusion (ICA terminus is involved as the occlusion site) and (B) non-T occlusion (ICA terminus is not involved as the occlusion site). ICA: internal carotid artery the anterior communicating artery ( $\mathrm{ACoA})$ is confirmed using angiography via the unaffected side (Fig. 2B). Anterograde ICA blood flow is blocked with the BGC on the affected side. Initially, thrombus retrieval is attempted via manual aspiration through the BGC (Fig. 2C). When it is not achieved, blockage with the BGC is continued and thrombectomy is performed using a stent retriever and/or aspiration device (Fig. 2D). Recanalization is confirmed using retrograde angiography via the contralateral carotid artery under blood flow blockage with the BGC on the affected side (Fig. 2E). At this point, aspiration is performed through the BGC to promote contrast medium reflux to the affected side. After confirming complete recanalization at the cervical portion to the terminus using retrograde angiography, BGC blockage is removed. Lastly, recanalization is confirmed using anterograde angiography through the BGC on the affected side (Fig. 2F).
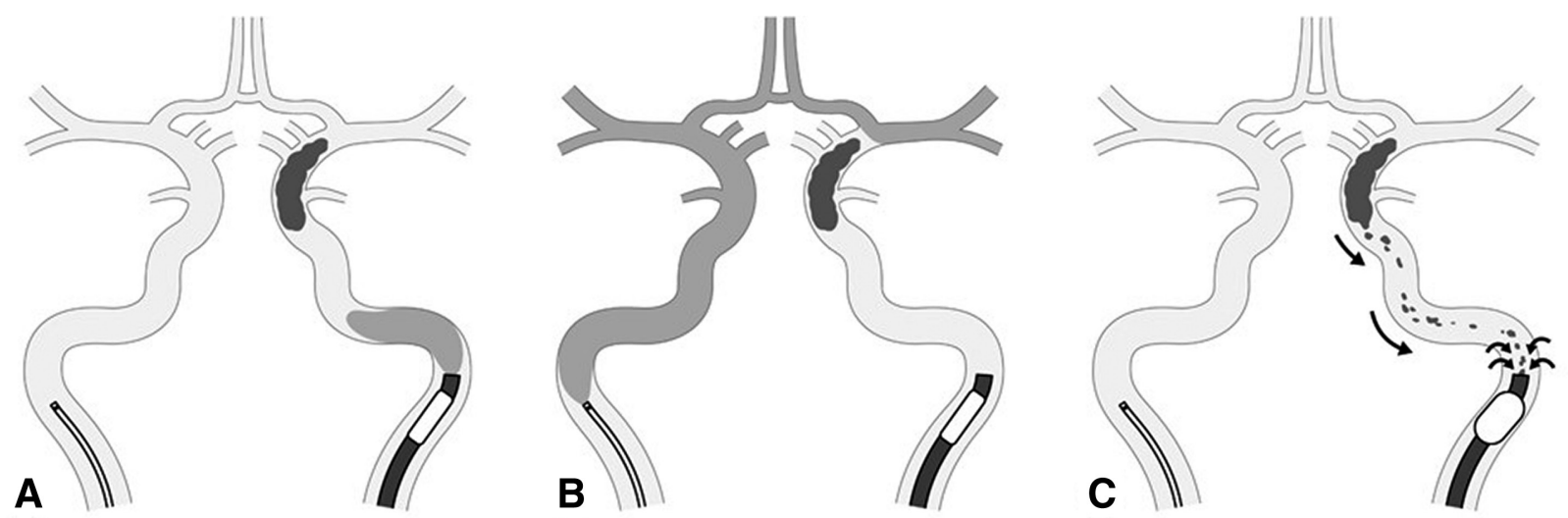
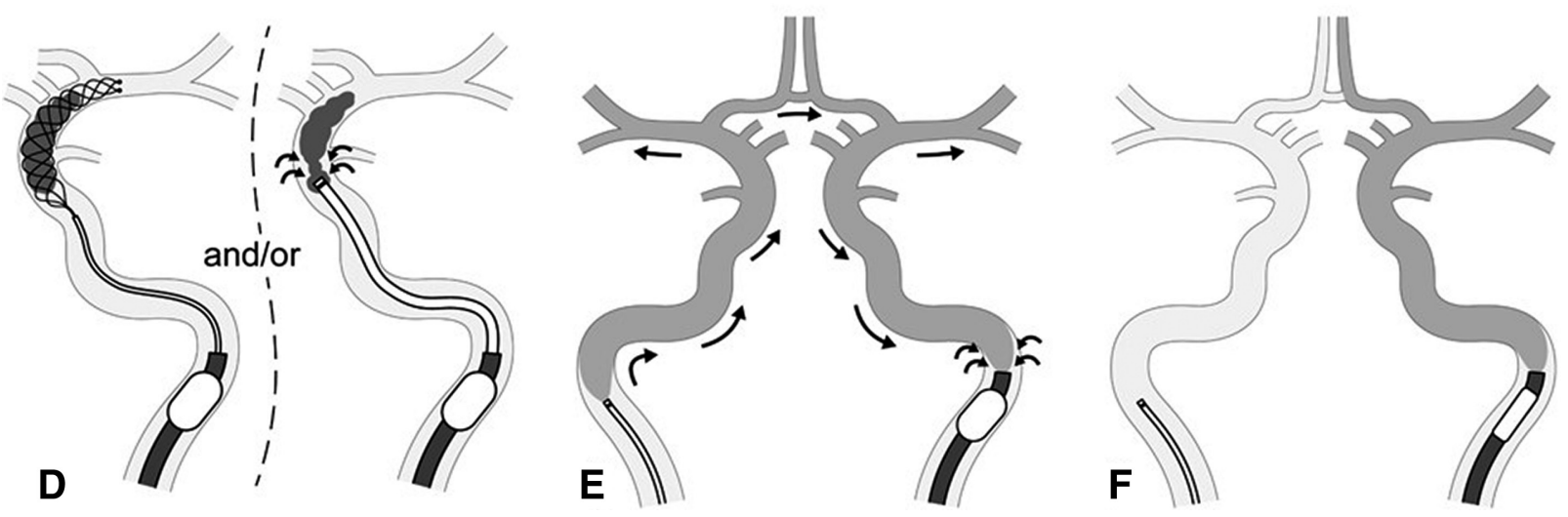

Fig. 2 Schematic illustration of the "dual puncture technique". (A) The BGC is navigated to the ICA at the affected side and another catheter is navigated to the unaffected side. Angiography via the BGC shows stagnation of the contrast media at the proximal ICA. (B) Angiography via the catheter at the unaffected side shows patency of the ACA and MCA at the affected side via the anterior communicating artery. (C) The ICA blood flow at the affected side is interrupted by the BGC. Initially, manual thrombus aspiration through the BGC is performed. (D) Subsequently, MT using a stent retriever and/or aspiration device is performed. (E) Reperfusion is confirmed by retrograde angiography via the carotid artery at the unaffected side with manual aspiration through the BGC. The blood flow of the ICA at the affected side is blocked by BGC until the confirmation of reperfusion. (F) Blockage of the ICA flow is removed. Lastly, reperfusion is confirmed by antegrade angiography via the BGC at the affected side. ACA: anterior cerebral artery; BGC: balloon guiding catheter; ICA: internal carotid artery; MCA: middle cerebral artery; MT: mechanical thrombectomy 
Three patients who were treated using this method are summarized in Table 1. The patient background and treatment procedures are described below.

\section{Case 1}

A 92-year-old woman developed left complete hemiplegia. Upon arrival, her National Institutes of Health Stroke Scale (NIHSS) score was 15. The Alberta Stroke Program Early CT score (ASPECTS)-diffusion-weighted imaging (DWI) on MRI was 9 (Fig. 3A and 3B). MRA revealed occlusion of the right ICA proximal to the terminus and right M1 segment of the MCA, suggesting a tandem lesion (Fig. 3C). ICA occlusion was evaluated as non-T occlusion. Emergency thrombectomy was performed. Initially, thrombectomy with the dual puncture technique for the right ICA occlusion was attempted to prevent distal embolization to the anterior cerebral artery (ACA) area. Bilateral femoral artery puncture was performed. Right common carotid angiography demonstrated contrast medium retention at the proximal area of the right ICA (Fig. 3D). On angiography via the left common carotid artery (CCA), bilateral ACAs were visualized (Fig. 3E). A FlowGate 8Fr (Stryker, Kalamazoo, MI, USA) was used to guide to the right ICA. After blocking the right ICA blood flow, a large volume of thrombus was retrieved by manual blood aspiration through the FlowGate (Fig. 3F). Retrograde angiography via the left CCA confirmed right ICA recanalization (31 min after the first puncture session) (Fig. 3G). Right ICA angiography through the $\mathrm{BGC}$ revealed that $\mathrm{M} 1$ occlusion remained. Thrombectomy using a combined technique with a Penumbra ACE 68 (Penumbra, Alameda, CA, USA) and Trevo Xp $4 \times 30 \mathrm{~mm}$ (Stryker), in addition to the arterial injection of urokinase, was added. Thrombolysis in cerebral infarction (TICI) grade 2a recanalization was achieved (Fig. $\mathbf{3} \mathbf{H}$ and 3I). The interval from the first puncture session to recanalization (fP2R) was 89 min. After 3 months, the patient's modified Rankin scale (mRS) score was 5.

\section{Case 2}

An 81-year-old man had reduced consciousness level, aphasia, and paralysis of the right upper and lower limbs. Upon arrival, his NIHSS score was 21. On MRI, the ASPECTS-DWI was 10 (Fig. 4A and 4B). MRA revealed occlusion proximal to the left ICA terminus (Fig. 4C), suggesting non-T occlusion. Emergency thrombectomy using the dual puncture technique was performed. The right femoral and right brachial arteries were punctured. The left femoral artery was occluded, and puncture was impossible. 

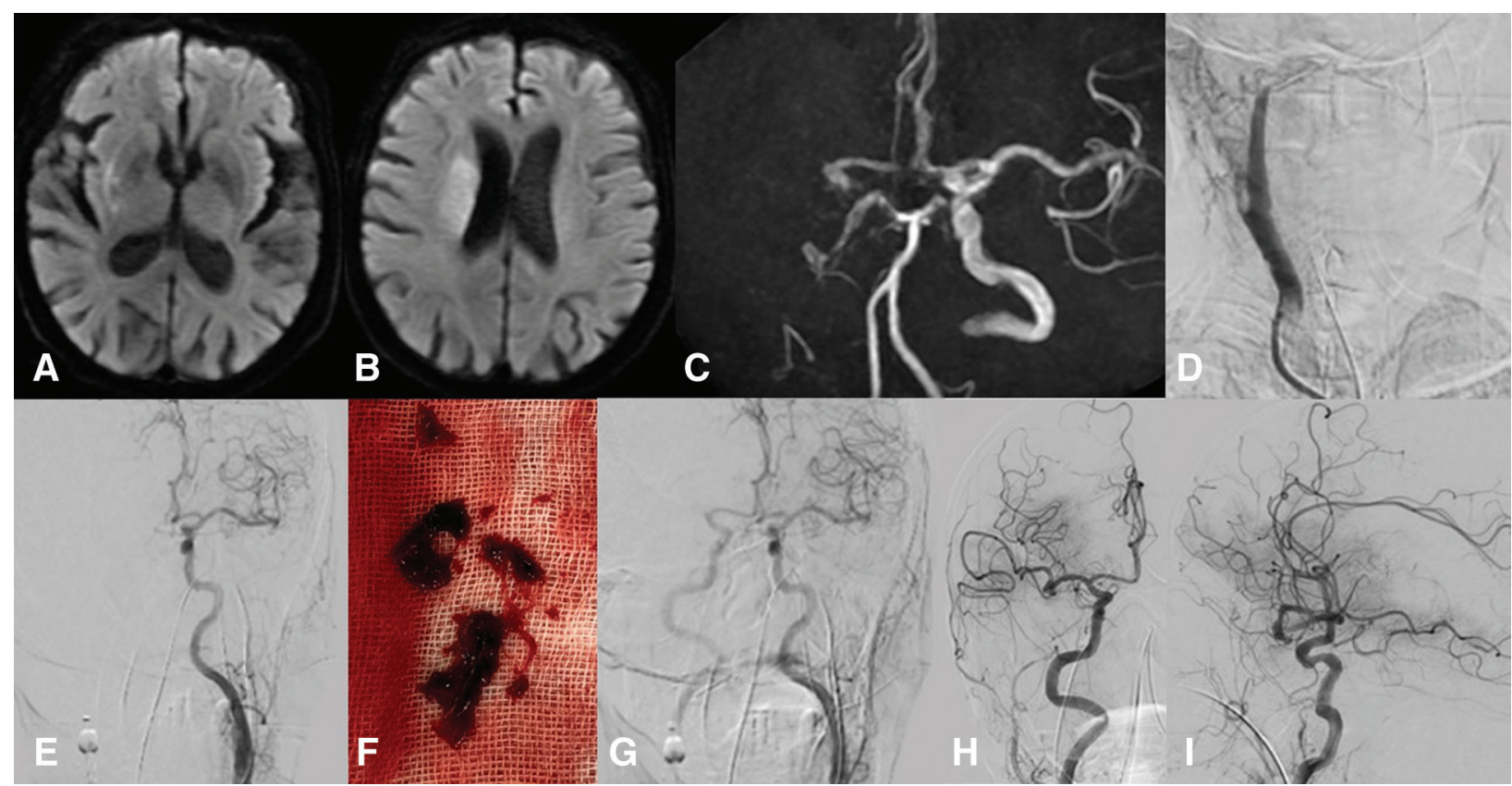

Fig. 3 Case 1. (A and B) The ASPECTS-DWI is 9 on MRI. (C) MRA shows occlusion of the right M1 and ICA with patency of the right ICA terminus, indicative of non-T occlusion. (D) Angiography of the right CCA shows stagnation of contrast media at the proximal ICA on the frontal projection. (E) Angiography of the left CCA reveals bilateral ACA. (F) The substantial thrombus is extracted by manual aspiration through the BGC. (G) Reperfusion of the right ICA is confirmed by retrograde angiography via the left CCA with manual aspiration through the BGC. ( $\mathbf{H}$ and $\mathbf{I})$ Antegrade angiography via the right ICA reveals $\mathrm{TICl}$ 2a recanalization on the frontal and lateral projections after thrombectomy for right MCA occlusion. ACA: anterior cerebral artery; ASPECTS-DWI; Alberta Stroke Program Early CT scorediffusion-weighted imaging; BGC: balloon guiding catheter; ICA: internal carotid artery; CCA: common carotid artery; MCA: middle cerebral artery; $\mathrm{TICl}$ : thrombolysis in cerebral infarction

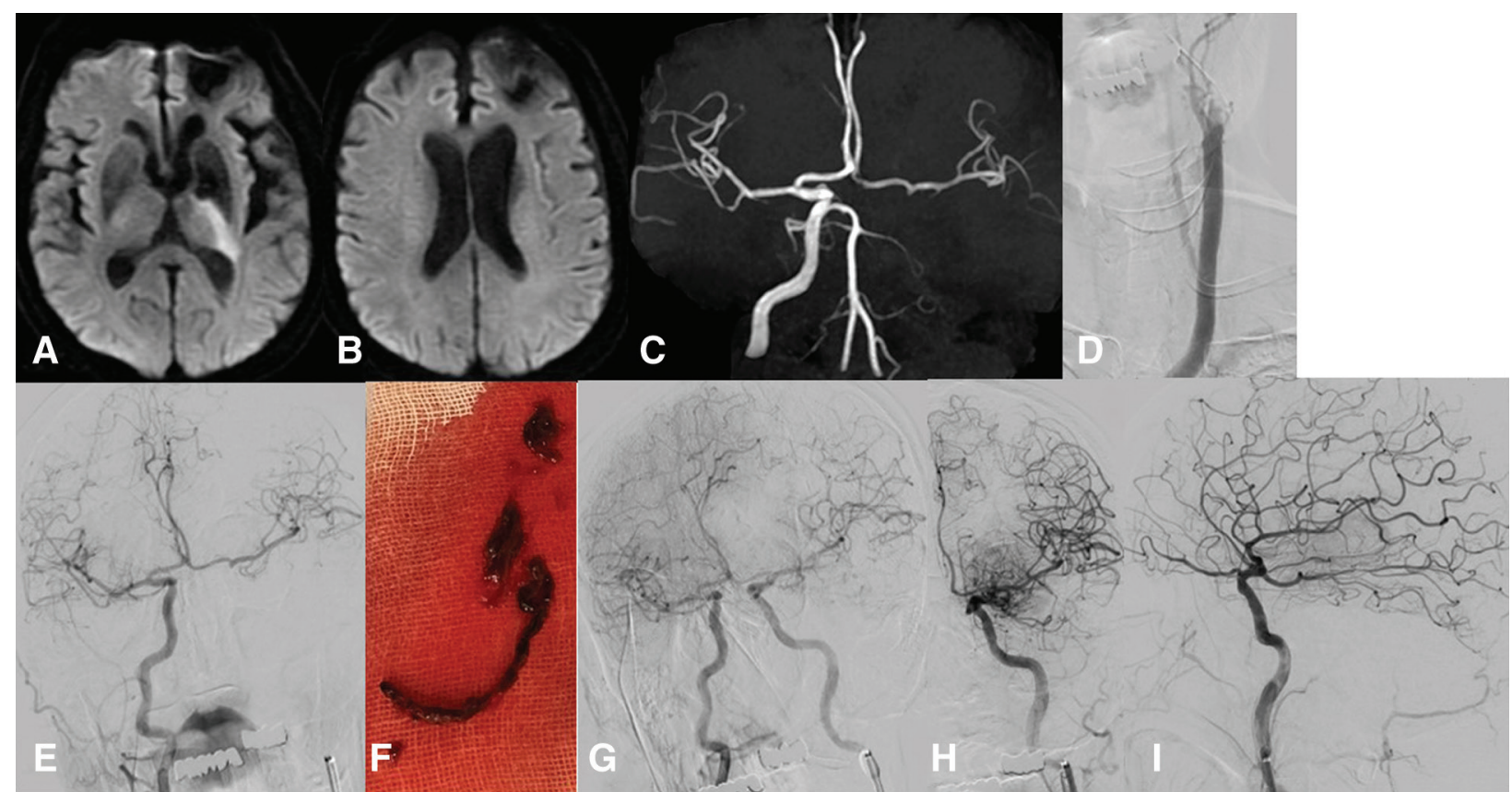

Fig. 4 Case 2. (A and B) The ASPECTS-DWI is 10 on MRI. (C) MRA shows left ICA occlusion with patency of the left ICA terminus, indicative of non-T occlusion. (D) Angiography of the right CCA shows proximal ICA occlusion on the frontal projection. (E) Angiography of the right CCA reveals the left ACA and MCA via collateral blood flow through the Acom. (F) The substantial thrombus is extracted by the ADAPT technique using a Penumbra ACE68. (G) Reperfusion of the left ICA is confirmed by retrograde angiography via the right CCA with manual aspiration through the BGC. (H and I) Antegrade angiography via the left ICA reveals $\mathrm{TICI} 3$ recanalization on the frontal and lateral projections. ACA: anterior cerebral artery; Acom: anterior communicating artery; ADAPT: a direct aspiration first pass technique; ASPECTS-DWI: Alberta Stroke Program Early CT score-diffusionweighted imaging; BGC: balloon guiding catheter; CCA: common carotid artery; ICA: internal carotid artery; MCA: middle cerebral artery; $\mathrm{TICl}$ : thrombolysis in cerebral infarction 
On the left CCA angiography, the area distal to the ICA origin was poorly visualized (Fig. 4D). An 8Fr FlowGate was inserted to the left ICA. Angiography through a catheter inserted into the right CCA via the right brachial artery demonstrated that an ACoA-mediated collateral pathway was responsible for blood supply to the left ACA and MCA (Fig. 4E). Thrombus retrieval was not achieved with manual aspiration through the BGC. Based on the microguidewire movement, the proximal end of the thrombus was considered to be present at the petrous portion of the ICA. A Penumbra ACE 68 was inserted to the petrous portion, retrieving a large volume of thrombus using the ADAPT technique $^{8)}$ (Fig. 4F). Retrograde angiography via the right carotid artery confirmed recanalization (Fig. 4G). Subsequently, angiography via the left side confirmed TICI grade 3 recanalization (Fig. $\mathbf{4 H}$ and $\mathbf{4 I}$ ). The $\mathrm{fP} 2 \mathrm{R}$ was 37 min. After 3 months, the patient's mRS score was 2 .

\section{Case 3}

A 70-year-old man had mild paralysis of the left upper limb. On the initial consultation, complete paralysis of the left upper limb, incomplete paralysis of the left lower limb, and left hemispatial neglect were observed. His NIHSS score was 11. MRI revealed multiple cerebral infarction of the right hemisphere. The ASPECTS-DWI was 11 (Fig. 5A and 5B). On MRA, the right ICA was interrupted at the cervix (Fig. 5C), suggesting non-T occlusion. Thrombectomy with the dual puncture technique via bilateral femoral artery puncture was performed. On right CCA angiography, the area distal to the ICA origin was poorly visualized (Fig. 5D). A Branchor 9Fr (Asahi Intecc, Aichi, Japan) was inserted to the right ICA. Angiography through a catheter inserted into the left ICA demonstrated that an ACoA-mediated collateral pathway was responsible for the blood supply to the right ACA and MCA (Fig. 5E). Thrombus retrieval was not achieved with manual aspiration through the BGC. Microguidewire movement suggested occlusion at the cavernous portion of the ICA. A Solitaire Platinum $6 \times 40 \mathrm{~mm}$ (Medtronic, Minneapolis, MN, USA) was deployed from the $\mathrm{C} 2$ to the cavernous portion of the ICA (Fig $\mathbf{5} \mathbf{F}$ and $\mathbf{5 G}$ ). Angiography of the left side confirmed retrograde flow restoration (Fig. 5H). A Penumbra JET7 (Penumbra) was inserted to the cavernous portion, and the Solitaire was retrieved into the Penumbra under the applied vacuum in the Penumbra using an aspiration pump (aspiration catheter with proximal balloon [ASAP] technique ${ }^{9)}$ ). Retrograde angiography via the left carotid artery confirmed recanalization (Fig. 5I), which was also subsequently confirmed by anterograde angiography at the right side. The fP2R was 36 min. However, blood flow was delayed, suggesting the presence of a dissecting lesion involving the right cervical to the cavernous portion (Fig. 5J). Two Carotid Wallstents (Boston Scientific, Natick, MA, USA) and a Neuroform Atlas (Stryker) were inserted, and the blood flow was improved (Fig 5K and $\mathbf{5 L}$ ). TICI grade 3 recanalization was achieved (Fig 5M and $\mathbf{5 N}$ ). After 3 months, his mRS score was 1 .

\section{Discussion}

Few comparative studies have investigated the differences between $\mathrm{T}$ occlusion and non-T occlusion in patients with acute ICA occlusion based on clinical features and treatment results of MT.710) These two factors may influence MT strategies, as ischemic tolerance depends on the presence of an ACoA-mediated contralateral collateral pathway. In $\mathrm{T}$ occlusion, the collateral pathway is poor and thus requires more prompt recanalization as compared with non-T occlusion. ${ }^{2,10,11)}$ Ota et al. ${ }^{7)}$ retrospectively examined 81 patients who underwent MT for T occlusion or non-T occlusion and reported results of their multicenter cooperative study. Participants included 61 patients with T occlusion and 20 with non-T occlusion. No significant differences were observed in terms of treatment outcomes or incidence of distal embolization as a complication between the two groups. However, in their study, MT procedures were not standardized, and the influence of different procedures on treatment outcomes was not investigated.

With regard to MT for non-T occlusion, if the ICA terminus or MCA is occluded due to distal embolization, neurological symptom may be markedly deteriorated; therefore, careful monitoring should be made to prevent distal embolization. To achieve this, anterograde blood flow blockage should be continued until the thrombus is completely removed. In addition, careless anterograde angiography via the affected side for confirming recanalization should be avoided. Using a BGC, ICA blockage during the procedure helps prevent the occurrence of distal embolization. ${ }^{12)}$ From this viewpoint, MT was performed for non- $\mathrm{T}$ occlusion using the dual puncture technique under anterograde ICA blood flow blockage with a BGC balloon from the first angiography session after BGC insertion to the confirmation of recanalization using retrograde angiography via the contralateral carotid artery. In our three patients, this method facilitated distal embolizationfree recanalization of the ICA. Tsuji et al. ${ }^{6}$ performed 


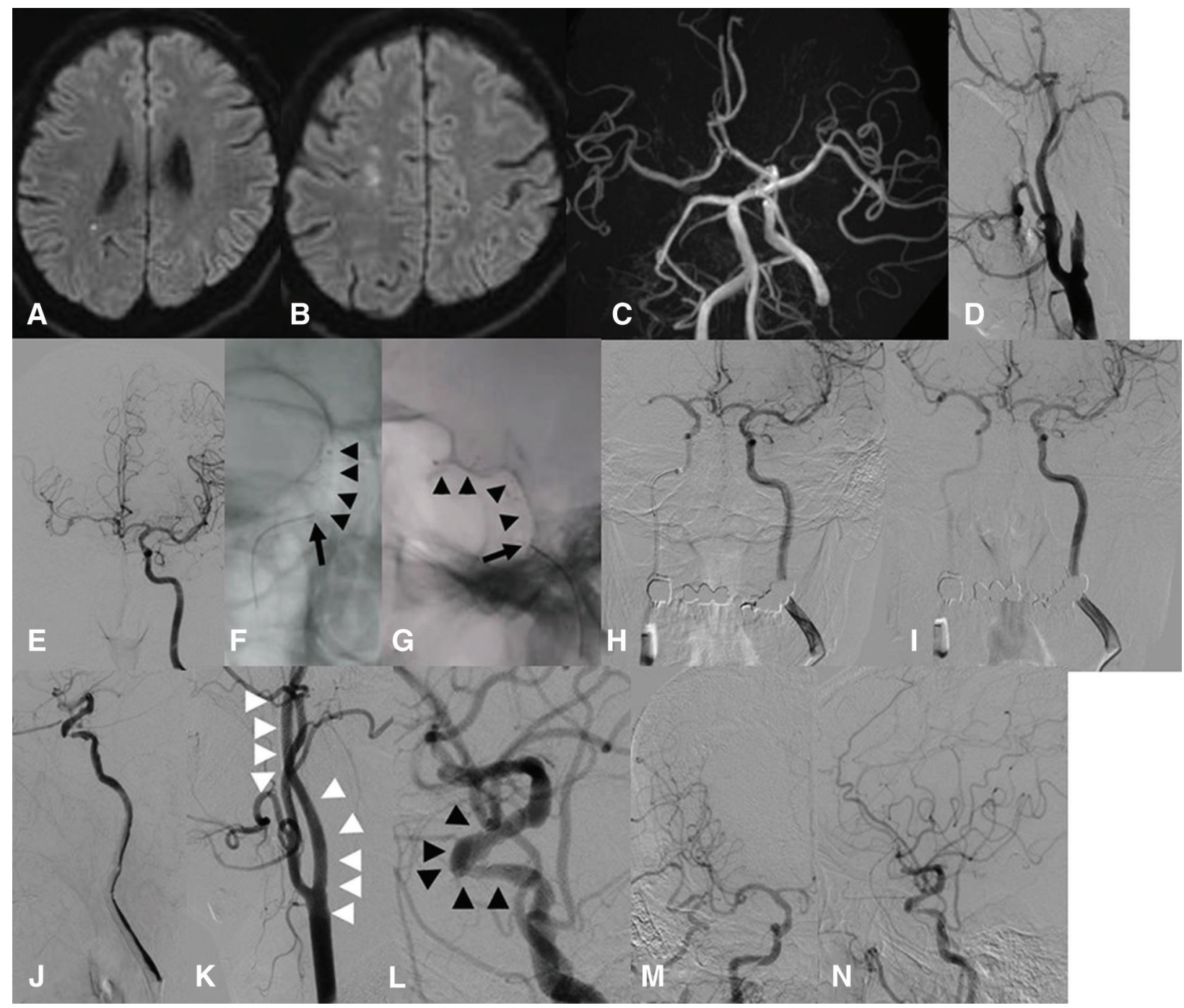

Fig. 5 Case 3. (A and B) MRI shows multiple DWI high lesions in the right hemisphere. (C) MRA shows right ICA occlusion with patency of the right ICA terminus, indicative of non-T occlusion. (D) Angiography of the right CCA shows stagnation of contrast media at the proximal ICA on the lateral projection. (E) Angiography of the left CCA reveals the right $\mathrm{ACA}$ and $\mathrm{MCA}$ via collateral blood flow through the Acom. (F and $\mathbf{G}$ ) MT by combined technique using a Solitaire platinum $6 \mathrm{~mm} \times 40 \mathrm{~mm}$ and Penumbra JET7 on the frontal and lateral projection. (Arrowheads: Solitaire, arrow: the tip of JET7). (H) Immediate flow restoration is confirmed by retrograde angiography via the left ICA with manual aspiration through the JET7. (I) Reperfusion of the right ICA is confirmed by retrograde angiography via

contralateral carotid angiography using a method similar to ours in six patients with acute ICA occlusion, including T occlusion, and reported its usefulness for the prevention of distal embolization.

In patients with non-T occlusion, the thrombus site is relatively proximal. In some cases, complete recanalization is relatively readily achieved with manual aspiration through a BGC; thus, it should be initially performed. In Case 1, a large volume of thrombus was retrieved by manual the left ICA with manual aspiration through the BGC. (J) Antegrade angiography via the right ICA reveals delayed lamina flow with a severe stenotic lesion at the cavernous portion of the ICA. ( $\mathbf{K}$ and $\mathbf{L}$ ) Additional treatment via placement of a Carotid Wallstent and Neuroform Atlas is performed. (White arrowheads: Wallstent, black arrowheads: Neuroform) ( $\mathbf{M}$ and $\mathbf{N}$ ) Final antegrade angiography via the right ICA reveals $\mathrm{TICI} 3$ recanalization on the frontal and lateral projections. ACA: anterior cerebral artery; Acom: anterior communicating artery; BGC: balloon guiding catheter; CCA: common carotid artery; DWI: diffusion-weighted imaging; ICA: internal carotid artery; MCA: middle cerebral artery; MT: mechanical thrombectomy; TICI: thrombolysis in cerebral infarction

aspiration through a $\mathrm{BGC}$ and ICA recanalization was promptly achieved. According to a report published by Ota et al., the incidences of intracranial ICA stenosis and arterial dissection in patients with non- $\mathrm{T}$ occlusion were significantly higher than in those with $\mathrm{T}$ occlusion ${ }^{7)}$; the necessity of additional treatment for these lesions after thrombectomy must also be considered. In Case 3, a dissecting lesion was suggested, and additional stenting was performed. In some patients with non-T occlusion, symptoms 
at the time of onset are relatively mild due to the presence of a collateral pathway ${ }^{13}$; therefore, physicians may hesitate to initiate therapeutic intervention with endovascular treatment. In Case 3, symptoms at the time of onset were mild and the patient presented to our department on foot. According to a study regarding balloon test occlusion of the ICA (intentionally prepared state of non-T occlusion), permanent blockage may induce delayed ischemic symptoms even if tolerance is evaluated as favorable based on clinical symptoms during examination. ${ }^{14}$ ) Physicians should not be misled with early mild symptoms after onset in order to not miss the timing of treatment.

The dual puncture technique is effective at preventing distal embolization, but the operative time is longer than that of the standard procedure. If arterial puncture at two sites and catheter guiding into the contralateral carotid artery can be smoothly performed, the time lost may be limited to a few minutes. However, if unexpected issues, such as the necessity of changing the site of brachial artery puncture, occur as demonstrated in Case 2, the time lost may increase. When performing MT for acute large vessel occlusion, a strong correlation was observed between the interval reduction until recanalization and improvement in the functional prognosis. ${ }^{15)} \mathrm{A}$ "trade-off" relationship was observed between the prevention of distal embolization and interval reduction until recanalization. In patients with $\mathrm{T}$ occlusion, prompt recanalization is required because the ischemic area is extensive, involving the ACA and MCA. ${ }^{2,10,11)}$ In patients with $\mathrm{T}$ occlusion, recanalization should be promptly achieved using the standard procedure. Conversely, during the recanalization therapy for non- $\mathrm{T}$ occlusion, a collateral pathway from the unaffected side to the MCA may be present, and thereby slight time prolongation is acceptable. In patients with non-T occlusion, distal embolization must be prevented even if the operative time is slightly prolonged; thus, the dual puncture technique may be positively applied. However, this does not apply to patients with serious neurological symptoms or those with a low ASPECTS. In the above-mentioned patients with non- $T$ occlusion, a reduction in the interval until recanalization should be prioritized, as indicated for patients with $\mathrm{T}$ occlusion.

\section{Conclusion}

MT for non-T occlusion may cause serious neurological symptoms due to distal embolization. The dual puncture technique may help prevent distal embolization through continuous anterograde blood flow blockage and recanalization confirmation on retrograde angiography via the unaffected side. In patients with non-T occlusion, the dual puncture technique with retrograde angiography via the contralateral carotid artery to prevent distal embolization should be positively applied even if the operative time is slightly prolonged.

\section{Acknowledgment}

I would like to thank Dr. Hiroshi Yamagami for useful discussions and advice, and Dr. Tatusya Mori for collaborating in the endovascular treatment.

\section{Disclosure Statement}

The authors declare no conflicts of interest.

\section{References}

1) Broderick JP, Palesch YY, Demchuk AM, et al: Endovascular therapy after intravenous t-PA versus t-PA alone for stroke. N Engl J Med 2013; 368: 893-903.

2) Fischer U, Mono ML, Schroth G, et al: Endovascular therapy in 201 patients with acute symptomatic occlusion of the internal carotid artery. Eur J Neurol 2013; 20: 1017-1024, e87.

3) Li W, Yin Q, Xu G, et al: Treatment strategies for acute ischemic stroke caused by carotid artery occlusion. Intervent Neurol 2016; 5: 148-156.

4) Lin R, Vora N, Zaidi S, et al: Mechanical approaches combined with intra-arterial pharmacological therapy are associated with higher recanalization rates than either intervention alone in revascularization of acute carotid terminus occlusion. Stroke 2009; 40: 2092-2097.

5) Paciaroni M, Balucani C, Agnelli G, et al: Systemic thrombolysis in patients with acute ischemic stroke and Internal Carotid ARtery Occlusion: the ICARO study. Stroke 2012; 43: $125-130$

6) Tsuji K, Nakagawa N, Fukawa N, et al: Usefulness of contralateral carotid angiography in revascularization therapy of acute internal carotid artery occlusion. J Neurosurg 2018; 129: 465-470.

7) Ota $T$, Shigeta $K$, Amano $T$, et al: Endovascular treatment for acute carotid $\mathrm{T}$ and carotid non- $\mathrm{T}$ occlusion: a retrospective multicentre study of 81 patients. Interv Neuroradiol 2019; 25: 497-501.

8) Turk AS, Spiotta A, Frei D, et al: Initial clinical experience with the ADAPT technique: a direct aspiration first pass technique for stroke thrombectomy. J Neurointerv Surg 2014; 6: 231-237. 
9) Goto $\mathrm{S}$, Ohshima T, Ishikawa $\mathrm{K}$, et al: A stent-retrieving into an aspiration catheter with proximal balloon (ASAP) technique: a technique of mechanical thrombectomy. World Neurosurg 2018; 109: e468-e475.

10) Liebeskind DS, Flint AC, Budzik RF, et al: Carotid I's, L's and T's: collaterals shape the outcome of intracranial carotid occlusion in acute ischemic stroke. $J$ Neurointerv Surg 2015; 7: 402-407.

11) Noh Y, Jung CK, Hong JH, et al: Recanalization rate and clinical outcome in acute carotid-T occlusion. Eur Neurol 2015; 74: 36-42.

12) Chueh JY, Kühn AL, Puri AS, et al: Reduction in distal emboli with proximal flow control during mechanical thrombectomy: a quantitative in vitro study. Stroke 2013; 44: 1396-1401.

13) Suzuki R, Ota T, Furuta $Y$ et al: Five cases of acute nonterminal internal carotid artery occlusion followed by delayed spontaneous recanalization. Jpn J Stroke 2019; 41: 499-504 (in Japanese).

14) Dare AO, Chaloupka JC, Putman CM: Failure of the hypotensive provocative test during temporary balloon test occlusion of the internal carotid artery to predict delayed hemodynamic ischemia after therapeutic carotid occlusion. Surg Neurol 1998; 50: 147-155; discussion 155-156.

15) Saver JL, Goyal M, van der Lugt A, et al: Time to treatment with endovascular thrombectomy and outcomes from ischemic stroke: a meta-analysis. JAMA 2016; 316: 1279-1288. 